\title{
Aeroacoustic computation of fluid-structure interaction prob- lems with low Mach numbers
}

\author{
Jan Valášek ${ }^{1,2, \star}$ and Petr Sváček ${ }^{1,2}$ \\ ${ }^{1}$ Department of Technical Mathematics, Faculty of Mechanical Engineering, Czech Technical University in Prague, Karlovo nám. \\ 13, Praha 2, 121 35, Czech Republic \\ ${ }^{2}$ Dept. of Instrumentation and Control Engineering, Faculty of Mechanical Engineering, Center of Advanced Aerospace Technol- \\ ogy, Czech Technical University in Prague, Technicka Street 4, 16607, Prague 6, Czech Republic
}

\begin{abstract}
This contribution deals with the acoustic simulation of aerodynamical noise generated by a flow over an airfoil or by flow in a flexible channel. Since the considered flow has low Mach number the hybrid approach of acoustic analogies can be applied here with benefits. The fluid-structure-acoustic interaction problem is generally described as a quite complicated problem comprising of three different physical fields - the vibration of the elastic body, the unsteady fluid flow and the acoustics together with mutual couplings. The fluid flow in time dependent domain is governed by the incompressible Navier-Stokes equations in arbitrary Langrangian-Eulerian formulation and the elastic structure is modelled by the means of linear elasticity theory. The Lighthill analogy and acoustic perturbation equation (APE) is considered to describe the sound propagation. The simulation of fluid-structure (FSI) interaction and acoustic field is implemented using the FEM in an in-house solver. The sound sources computed from FSI results are analyzed and within sound propagation simulation the perfectly matched layer technique is used. In the end the results of Lighthill and APE analogy are compared.
\end{abstract}

\section{INTRODUCTION}

The simulation of noise creation and propagation is of a quite big importance today due to increasing number and power of noise producing devices. Good examples of external and internal problems with aerodynamically generated sound are air flow around airfoil, air-conditioning or sound produced by human vocal fold model. These problems are studied by experimental, analytical and numerical methods in many applications, see for example [1] or [2], nevertheless the acoustic simulation within fluid-structure interaction (FSI) problem is used only rarely, see for example [3].

The acoustic quantities can be possibly obtained from solution of compressible Navier-Stokes equations and by later filtering of acoustic parts but such an approach is extremely computational demanding if not infeasible in many cases. That is why usually simplified approaches are used. The development of these approaches, called acoustic analogies, started by Lighthill in 1952, see [4]. Then the new idea of acoustic/viscous splitting were introduced in 1994 by Hardin and Pope [5] and the cornerstone of modern computational aeroacoustics was laid by Ewert and Schröder in 2003, see [6]. They derived new system of equations from Navier-Stokes equations by filtering based on splitting flow variables in three parts. Here, we focus on

\footnotetext{
^e-mail: jan.valasek1@fs.cvut.cz
}

the acoustic perturbation equation (APE) analogy and also the well-known Lighthill analogy is explained and used to verify the results of APE analogy.

In general the fluid-structure-acoustic interaction (FSAI) problem, see e.g. [2], is very complex phenomenon composed of three different physical fields - the deformation of the elastic structure, the unsteady fluid flow and the acoustics together with mutual couplings. The hybrid approach of acoustic analogies allows to decouple fluid-structure interaction (FSI) problem and acoustics, i.e. the effects of acoustic coupling to FSI problem is neglected due to the small intensity of resulting acoustic field. It enables us to solve acoustic problem in parallel or at the end of FSI computation, where from the stored fluid velocities and pressures the sound source is calculated. Moreover the interpolation onto (usually) coarser acoustic grid, [7], can be performed to save the computational time. During solution of sound propagation the perfectly matched layer (PML) technique is used due to the simulation in the open domains. This additional layer of elements introduces effective damping of outgoing waves without reflection at the interface between PML and acoustic propagation domain, [8].

The fluid flow is governed by the incompressible Navier-Stokes equations with the aid of the Arbitrary Lagrangian-Eulerian (ALE) method, which allows the computational domain of the fluid to be deformed. The 
elastic structure is modelled by the means of linear elasticity theory, where material with more layers is used. All the three subproblems are numerically approximated by the finite element method. Specially, the fluid flow simulation is stabilized by the modified Streamline-Upwind/PetrovGalerkin stabilization, see [9].

In the end the numerical simulation of sound propagation is given and the results of the Lighthill analogy and APE analogy is presented.

\section{MATHEMATICAL DESCRIPTION}

For the sake of simplicity only a two-dimensional problem is described here. The FSI problem is solved on a small subdomain of the whole domain, where the sound propagation problem is considered. The scheme of FSI domain is shown in Figure 1 and the whole computational domain for acoustic problem is depicted in Figure 2. The FSI domain consists of an elastic body domain $\Omega^{s}$ and a fluid domain $\Omega^{f}$, where the sound sources are computed from the flow quantities. The elastic structure is described in Lagrange coordinate system, therefore $\Omega_{\text {ref }}^{s}$ denotes the reference state, which remains the same for any time $t$. For the description of the fluid flow on moving domain $\Omega_{t}^{f}$ the ALE method is used and we distinguish between the reference domain $\Omega_{\text {ref }}^{f}$ at time instant $t=0$ and the deformed domain $\Omega_{t}^{f}$ at arbitrary time $t$. Similarly, by $\Gamma_{\mathrm{W}_{\text {ref }}}$ and $\Gamma_{\mathrm{W}_{\mathrm{t}}}$ we denote the reference state and deformed state at arbitrary time $t$ of the interface, respectively.

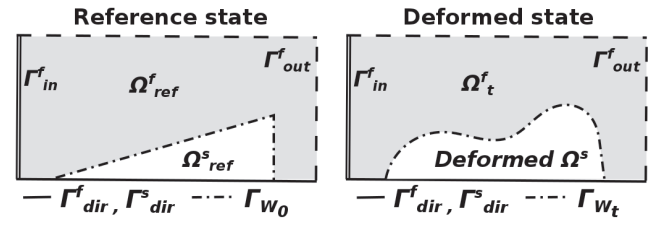

Fig. 1. Scheme of FSI domain consisting of elastic structure domain and fluid domain marked before (left) and after (right) a deformation.

\subsection{ALE method}

The ALE method enables to describe the fluid flow in a time-dependent domain. The basis of this method lies in the construction of a diffeomorphism $A_{t}$ which maps the reference (undistorted) domain $\Omega_{\text {ref }}^{f}$ on to the domain $\Omega_{t}^{f}$ at any instant time $t \in(0, \mathrm{~T})$. Under assumption of continuity of the time derivative $\frac{\partial A_{t}}{\partial t} \in C\left(\overline{\Omega_{\text {ref }}^{f}}\right)$ the ALE domain velocity $\mathbf{w}_{D}$ can be defined as

$$
\mathbf{w}_{D}\left(A_{t}(X), t\right)=\frac{\partial}{\partial t} A_{t}(X), \quad t \in(0, \mathrm{~T}), X \in \Omega_{\mathrm{ref}}^{f} .
$$

Finally, the ALE derivative is introduced as the time derivative with respect to a fixed point $X \in \Omega_{r e f}^{f}$. The ALE derivative can be expressed as

$$
\frac{D^{A}}{D t} f(x, t)=\frac{\partial f}{\partial t}(x, t)+\mathbf{w}_{D}(x, t) \cdot \nabla f(x, t) .
$$

More details and practical construction of ALE mapping is described e.g. in [10] or [11].

\subsection{Fluid flow}

The flow of the viscous incompressible fluid in time variable domain $\Omega_{t}^{f}$ obeys the Navier-Stokes equations in the ALE form

$\frac{D^{A} \mathbf{v}}{D t}+\left(\left(\mathbf{v}-\mathbf{w}_{D}\right) \cdot \nabla\right) \mathbf{v}-v^{f} \Delta \mathbf{v}+\nabla p=\mathbf{0}, \quad \operatorname{div} \mathbf{v}=0$ in $\Omega_{t}^{f}$,

where $\mathbf{v}(x, t)$ is the fluid velocity, $p$ denotes the kinematic pressure and $v^{f}$ is the kinematic fluid viscosity. Equations (3) are supplied by the zero initial conditions and following boundary conditions. For $x \in \Gamma_{\text {Dir }}^{f} \cup \Gamma_{\mathrm{W}_{\mathrm{t}}}$ and $t \in(0, \mathrm{~T})$ holds

$$
\mathbf{v}(x, t)=\mathbf{w}_{D}(x, t),
$$

and the pressure fulfills for $x \in \Gamma_{\text {In }}^{f} \cup \Gamma_{\text {Out }}^{f}, t \in(0, T)$

$$
p(x, t) \mathbf{n}^{f}-v^{f} \frac{\partial \mathbf{v}}{\partial \mathbf{n}^{f}}(x, t)=-\frac{1}{2} \mathbf{v}\left(\mathbf{v} \cdot \mathbf{n}^{f}\right)^{-}+p_{\text {ref }} \mathbf{n}^{f},
$$

where $\mathbf{n}^{f}$ is unit outer normal to boundary $\partial \Omega_{t}^{f}$ and $p_{\text {ref }}$ is a reference pressure, which has possibly different values at the inlet $\Gamma_{\text {In }}^{f}$ and at the outlet $\Gamma_{\text {Out }}^{f}$. The condition (5) is the modified do-nothing boundary condition, which suppresses the possible backward inlet through the outlet part of the boundary, see [12].

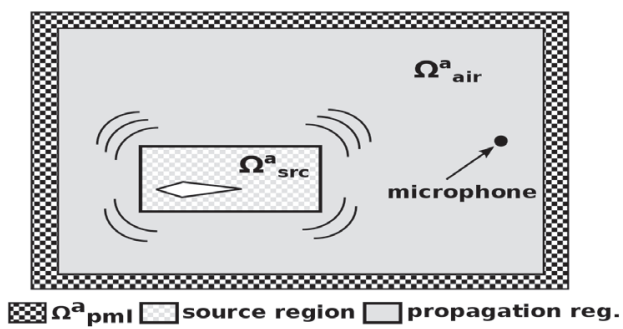

Fig. 2. Scheme of acoustic domain. The domain, where the sound propagation is computed, is composed of the sound source region $\Omega_{\mathrm{src}}^{a}$ (equals FSI domain without structure domain, i.e. it equals reference fluid domain $\Omega_{\text {ref }}^{f}$ ) and the propagation region. It is closed by the PML blocks.

\subsection{Elastic body}

The deformation $\mathbf{u}(X, t)=\left(u_{1}, u_{2}\right)$ of the elastic body $\Omega^{s}$ is modelled by the partial differential equations

$$
\rho^{s} \frac{\partial^{2} u_{i}}{\partial t^{2}}-\frac{\partial \tau_{i j}^{s}(\mathbf{u})}{\partial X_{j}}=f_{i}^{s} \quad \text { in } \Omega^{s} \times(0, \mathrm{~T}),
$$

where $\rho^{s}$ denotes the structure density, the tensor $\tau_{i j}^{s}$ is the Cauchy stress tensor, the vector $\mathbf{f}^{s}=\left(f_{1}^{s}, f_{2}^{s}\right)$ is the volume density of an acting force and $X=\left(X_{1}, X_{2}\right)$ are the reference coordinates. In the case of isotropic material the Hook law reads

$$
\tau_{i j}^{s}=\lambda^{s}(\operatorname{div} \mathbf{u}) \delta_{i j}+2 \mu^{s} e_{i j}^{s}(\mathbf{u}),
$$

where $e_{i j}^{s}$ are components of the strain tensor of small displacements $e^{s}(\mathbf{u})=\frac{1}{2}\left(\frac{\partial u_{j}}{\partial X_{k}}+\frac{\partial u_{k}}{\partial X_{j}}\right), \mathbb{I}=\left(\delta_{i j}\right)$ is the Kronecker's delta and $\lambda^{s}, \mu^{s}$ are Lamé coefficients. They possibly can have different values for different parts of the 
body. The equations (6) are supplied by the initial and boundary conditions

$$
\begin{gathered}
\text { a) } \mathbf{u}(X, 0)=\mathbf{u}_{0}(X), \frac{\partial \mathbf{u}}{\partial t}(X, 0)=\mathbf{u}_{1}(X), \quad \text { for } X \in \Omega^{s}, \\
\text { b) } \mathbf{u}(X, t)=\mathbf{u}_{\mathrm{Dir}}(X, t), \quad \text { for } X \in \Gamma_{\mathrm{Dir}}^{s}, t \in(0, \mathrm{~T}),(8) \\
\text { c) } \tau_{i j}^{s}(X, t) n_{j}^{s}(X)=q_{i}^{s}(X, t), \text { for } X \in \Gamma_{\mathrm{W}_{\mathrm{ref}}}^{s}, t \in(0, \mathrm{~T}),
\end{gathered}
$$

where the $\Gamma_{\mathrm{W}_{\text {ref }}}, \Gamma_{\text {Dir }}^{s}$ are mutually disjoint parts of the boundary $\partial \Omega^{s}=\Gamma_{\mathrm{W}_{\text {ref }}} \cup \Gamma_{\text {Dir }}^{s}$ (see Figure 1 ) and $n_{j}^{s}(X)$ are the components of the unit outer normal to $\Gamma_{\mathrm{W}_{\text {ref }}}$.

\subsection{Aeroacoustics}

The acoustic domain $\Omega^{a}$ is composed of three subdomains $\Omega_{\mathrm{src}}^{a}, \Omega_{\text {air }}^{a}$ and $\Omega_{\mathrm{pml}}^{a}$, see Figure 2. The domain $\Omega_{\mathrm{src}}^{a}$ of acoustic sources is the same as the reference fluid domain and sound sources are here evaluated. The domain $\Omega_{\text {air }}^{a}$ represents an air region without sound sources, where acoustic waves propagate freely. The both domains $\Omega_{\mathrm{src}}^{a}, \Omega_{\text {air }}^{a}$ of free sound propagation are enclosed by PML domain $\Omega_{\mathrm{pml}}^{a}$ to damp the outgoing sound waves.

The first considered analogy is the Lighthill acoustic analogy proposed in 1952, which has the form of the inhomogeneous wave equation

$$
\frac{1}{c_{0}^{2}} \frac{\partial^{2} p^{\prime}}{\partial t^{2}}-\frac{\partial^{2} p^{\prime}}{\partial x_{i}^{2}}=\frac{\partial^{2} T_{i j}}{\partial x_{i} \partial x_{j}},
$$

for unknown pressure fluctuation $p^{\prime}=p-p_{0}$ and with a given speed of sound $c_{0}$. It is derived from supposition of sound propagating from a small source region with fluid flow through a voluminous medium in the rest state, see [4]. Hence the Lighthill tensor $T_{i j}$ can be expressed as (hypothetical) stress difference between the flow and the rest state of the fluid

$$
T_{i j}=\rho^{f} v_{i} v_{j}+\left(\left(p-p_{0}\right)-c_{0}^{2}\left(\rho^{f}-\rho_{0}^{f}\right)\right) \delta_{i j}-\tau_{i j}^{f} \approx \rho^{f} v_{i} v_{j},
$$

where $p_{0}, \rho_{0}^{f}$ denotes the pressure and the density of the fluid at rest state, respectively. The used approximation in equation (10) for small Mach numbers was proposed already in [4]. The wave equation (9) is equipped with the zero initial condition and the boundary of acoustic domain $\partial \Omega^{a}$ with the outer unit normal $\mathbf{n}^{a}$ is considered as sound hard

$$
\frac{\partial p^{\prime}}{\partial \mathbf{n}^{a}}(x, t)=0 \quad \text { for } x \in \partial \Omega^{a}, t \in(0, \mathrm{~T}),
$$

which means that sound waves are there reflected back to the acoustic domain $\Omega^{a}$. The Lighthill analogy is quite easy to understand and it constitutes very general approach. It's disadvantage is that the pressure fluctuations $p^{\prime}$ can be considered as purely acoustic pressure $p_{a}$ only outside the fluid flow region, where no additional pressure is present.

The second presented acoustic analogy is the APE. It is analogy based on the splitting of physical quantities into the mean part $\overline{\mathbf{v}}, \bar{p}$, the fluctuating non-acoustic part $\mathbf{v}_{i c}, p_{i c}$ and the acoustic part $\mathbf{v}_{a}, p_{a}$

$$
\mathbf{v}=\overline{\mathbf{v}}+\mathbf{v}_{i c}+\mathbf{v}_{a}, \quad p=\bar{p}+p_{i c}+p_{a} .
$$

Inserting these expressions into the Navier-Stokes equations of incompressible fluid together with assumptions of the irrotational acoustic field $\left(\nabla \times \mathbf{v}_{a}=\mathbf{0}\right)$, isentropic flow, incompressible fluid $\left(\nabla \times \mathbf{v}_{i c}=0\right)$ and neglecting nonlinear terms results into system of equations

$$
\begin{aligned}
\frac{\partial p^{a}}{\partial t}+\overline{\mathbf{v}} \cdot \nabla p^{a}+\rho_{0}^{f} c_{0}^{2} \nabla \cdot \mathbf{v}^{a} & =-\frac{D p_{i c}}{D t}, \\
\frac{\partial \mathbf{v}^{a}}{\partial t}+\nabla\left(\overline{\mathbf{v}} \cdot \mathbf{v}^{a}\right)+\frac{1}{\rho_{0}^{f}} \nabla p^{a} & =\mathbf{0} .
\end{aligned}
$$

For the precise derivation and the discussion see [13]. This system can be further simplified into one scalar equation by introduction of the acoustic potential $\psi^{a}$ related to the acoustic particle velocity by $\mathbf{v}^{a}=-\nabla \psi^{a}$.

$$
\frac{1}{c_{0}^{2}} \frac{D^{2} \psi^{a}}{D t^{2}}-\Delta \psi^{a}=-\frac{1}{c_{0}^{2}} \frac{D p_{i c}}{D t},
$$

where $p_{i c}$ denotes the pressure connected with incompressible flow and the substantial derivative equals $\frac{D}{D t}=$ $\frac{\partial}{\partial t}+\overline{\mathbf{v}} \cdot \nabla$. For small velocities the mean velocity can be neglected $(\overline{\mathbf{v}}=0)$ and formulation (14) get again the form of inhomogenous wave equation

$$
\frac{1}{c_{0}^{2}} \frac{\partial^{2} \psi^{a}}{\partial t^{2}}-\Delta \psi^{a}=-\frac{1}{\rho_{0}^{f} c_{0}^{2}} \frac{\partial p_{i c}}{\partial t} .
$$

Equation (15) is called acoustic perturbation equation (APE).

Equation (14) or (15) is considered again with sound hard boundaries $\frac{\partial \psi^{a}}{\partial \mathbf{n}^{a}}(x, t)=0, x \in \partial \Omega^{a}, t \in(0, \mathrm{~T})$. The analogies based on the quantity splitting approach is physically more complicated than Lighthill analogy, on the other hand it delivers directly the acoustic pressure (through relation $p^{a}=\rho_{0}^{f} \frac{\partial \psi^{a}}{\partial t}$ ) in the whole acoustic domain.

\subsection{Coupling conditions}

The FSI problem is a coupled problem, where the location of the interface $\Gamma_{W_{t}}$ at any time $t$ depends on the solution of the FSI problem itself. It corresponds to the establishing force equilibrium between the aerodynamic and the elastic forces and it is implicitly given by deformation of the interface $\Gamma_{W_{\text {ref }}}$.

Moreover the requirement of stress continuity across the interface leads to the Neumann boundary condition (8 c) for the elastic problem in the form

$$
q_{i}^{s}(X, t)=-\sum_{j=1}^{2} \sigma_{i j}^{f}(x) n_{j}^{f}(x),
$$

where $x=X+\mathbf{u}(X, t)$ for any $X \in \Gamma_{\mathrm{W}_{\text {ref }}}$. The components of the fluid stress tensor are $\sigma_{i j}^{f}=-\rho_{0}^{f} p \delta_{i j}+\rho_{0}^{f} \nu^{f}\left(\frac{\partial v_{i}}{\partial x_{j}}+\frac{\partial v_{j}}{\partial x_{i}}\right)$ for $i, j \in\{1,2\}$.

The Dirichlet boundary condition postulated by equation (4) for the fluid flow problem is originated by the enforcement of velocity continuity across the interface.

Since the acoustic field has substantially less energy than the flow field, the backward coupling of acoustics to the flow field is not considered. 


\section{NUMERICAL SCHEME}

All three subproblems of FSAI problem is solved by finite element method. Therefore partial differential equations (6), (3) and (9) or (15) are reformulated in a weak sense in space and then this semi-discrete system is discretized in time by the finite difference scheme with the equidistant time step $\Delta t=\frac{T}{N}, N>>1$.

\subsection{Elastic body}

First, equation (6) is multiplied by a test function $\psi$ and integrated over the whole domain $\Omega^{s}$. The application of the Green theorem and Hooke law (7) leads to the classical weak form of elastic problem

$$
\begin{aligned}
\left(\rho^{s} \frac{\partial^{2} \mathbf{u}}{\partial t^{2}}, \psi\right)_{\Omega^{s}}+ & \left(\lambda^{s}(\operatorname{div} \mathbf{u}) \mathbb{I}+2 \mu^{s} \mathbf{e}^{\mathbf{s}}(\mathbf{u}), \mathbf{e}^{\mathbf{s}}(\psi)\right)_{\Omega^{s}}= \\
& =\left(\mathbf{f}^{s}, \psi\right)_{\Omega^{s}}+\left(\mathbf{q}^{s}, \psi\right)_{\Gamma_{\text {Neu }}^{s}}
\end{aligned}
$$

which needs to be fulfilled by all $\psi \in \mathbf{V}$. We use the notation $(\cdot, \cdot)_{\mathcal{D}}$ for the scalar product of the Lebesque spaces $L^{2}(\mathcal{D})$ or $\mathbf{L}^{2}(\mathcal{D})$. The space $\mathbf{V}=V \times V$, where

$$
V=\left\{\phi \in W^{1,2}\left(\Omega^{s}\right) \mid \phi=0 \text { on } \Gamma_{\text {Dir }}^{s}\right\},
$$

and $W^{1,2}(\Omega)$ is the Sobolev space, see [14]. The approximative solution $\mathbf{u}_{h}$ is sought in a finite element (FE) space, where it can be expressed as

$$
\mathbf{u}_{h}(x, t)=\sum_{j=1}^{N_{h}} \alpha_{j}(t) \boldsymbol{\psi}_{j}(x),
$$

where $\psi_{1}, \ldots, \psi_{N_{h}}$ are basis functions of FE space. Substituting (19) into (17) and setting $\psi=\psi_{i}$ gives the system of ordinary differential equations of second order for the unknown coeffients $\alpha_{j}(t)$

$$
\mathbb{M}^{T} \ddot{\alpha}+\mathbb{C}^{T} \dot{\alpha}+\mathbb{K}^{T} \alpha=\mathbf{b}(t),
$$

where the matrix $\mathbb{C}=\epsilon_{1} \mathbb{M}+\epsilon_{2} \mathbb{K}$ were added as the proportional damping with a proper choice of parameters $\epsilon_{1}, \epsilon_{2}$. The vector $\mathbf{b}(t)$ has components $b_{i}(t)=\left(\mathbf{f}^{s}, \boldsymbol{\psi}_{i}\right)_{\Omega^{s}}+$ $\left(\mathbf{q}^{s}, \boldsymbol{\psi}_{i}\right)_{\Gamma_{\text {Neu }}^{s}}$ and the elements of matrices $\mathbb{M}=\left(m_{i j}\right), \mathbb{K}=$ $\left(k_{i j}\right)$ are given by

$m_{i j}=\left(\rho^{s} \boldsymbol{\psi}_{j}, \boldsymbol{\psi}_{i}\right)_{\Omega^{s}}, k_{i j}=\left(\lambda^{s}\left(\operatorname{div} \boldsymbol{\psi}_{j}\right) \mathbb{I}+2 \mu^{s} \mathbf{e}^{s}\left(\boldsymbol{\psi}_{j}\right), \mathbf{e}^{s}\left(\boldsymbol{\psi}_{i}\right)\right)_{\Omega^{s}}$

System (18) is numerically discretized in time by the Newmark method, see for example [15] or [10].

\subsection{Fluid flow}

The discretization procedure of equation (3) is reverse - it is firstly discretized in time and after then in space. The ALE derivative is approximated by the BDF2 scheme

$$
\frac{D^{A} \mathbf{v}}{D t}\left(t_{n+1}\right) \approx \frac{3 \mathbf{v}^{n+1}-4 \overline{\mathbf{v}}^{n}+\overline{\mathbf{v}}^{n-1}}{2 \Delta t},
$$

where the notation $\overline{\mathbf{v}}^{i}(x)=\mathbf{v}^{i}(\tilde{x})$ for $\tilde{x}=A_{t_{i}}\left(A_{t_{n+1}}^{-1}(x)\right), i \in$ $\{n-1, n\}, x \in \Omega_{n+1}^{f}$ for a fixed time $t_{n+1}$ was used. In the rest of paper we omit the upper time index ${ }^{n+1}$ and set $\Omega^{f}:=\Omega_{t_{n+1}}^{f}$.

The similar steps as in the previous paragraph leads to the weak spatial formulation of equation (3) in time $t_{n+1}$. It can written as the abstract problem of seeking a function pair $V=(\mathbf{v}, p)$ such, that

$$
a(V, \boldsymbol{\Phi})=f(\boldsymbol{\Phi}),
$$

is satisfied for any test function $\boldsymbol{\Phi}=(\varphi, q)$ from space $\mathbf{X} \times M$. The space $M=L^{2}\left(\Omega^{f}\right)$ and $\mathbf{X}=X \times X$ is given as $X=\left\{f \in W^{1,2}\left(\Omega^{f}\right) \mid f=0\right.$ on $\left.\Gamma_{\mathrm{Dir}}^{f} \cup \Gamma_{\mathrm{In}}^{f} \cup \Gamma_{\mathrm{W}_{\mathrm{t}+1}}^{f}\right\} \subset W^{1,2}\left(\Omega^{f}\right)$

The form $a(\cdot, \cdot)$ is defined by

$$
\begin{aligned}
a(V, \boldsymbol{\Phi}) & =\left(\frac{3 \mathbf{v}}{2 \Delta t}, \varphi\right)_{\Omega^{f}}+\frac{1}{2}\left(\left(\left(\mathbf{v}-2 \mathbf{w}_{D}^{n+1}\right) \cdot \nabla\right) \mathbf{v}, \boldsymbol{\varphi}\right)_{\Omega^{f}}- \\
& -\frac{1}{2}((\mathbf{v} \cdot \nabla) \varphi, \mathbf{v})_{\Omega^{f}}+\frac{1}{2}\left((\mathbf{v} \cdot \mathbf{n})^{+} \mathbf{v}, \varphi\right)_{\Gamma_{\text {Out }}^{f}} \\
& +v^{f}(\nabla \mathbf{v}, \nabla \varphi)_{\Omega^{f}}-(p, \operatorname{div} \varphi)_{\Omega^{f}}+(q, \operatorname{div} \mathbf{v})_{\Omega^{f}}
\end{aligned}
$$

and functional $f(\boldsymbol{\Phi})=\left(\frac{4 \overline{\mathbf{v}}^{n}-\overline{\mathbf{v}}^{n-1}}{2 \Delta t}, \boldsymbol{\varphi}\right)_{\Omega^{f}}$. The nonlinear system of equations (23) is solved by fixed point iteration.

This formulation was further stabilized by the streamline-upwind/Petrov-Galerkin (SUPG) together with 'div-div' stabilization and the pressurestabilization/Petrov-Galerkin

(PSPG) in order to effectively decrease/reduce numerical instabilities associated with high Reynolds numbers. For details see [16] or [10].

\subsection{Coupling algorithm of FSI problem}

The advantage of hybrid approach is that FSI problem can be computed independently on the later acoustic simulation. Only the fluid pressure and velocities are needed to be saved for subsequent computing of acoustic sources given by (29) or (30). Then the relevant wave equation is solved.

The algorithm solving FSI problem is implemented in the form of strong coupling, see e.g. [11].

\subsection{Aeroacoustics}

In the case of Lighthill analogy, equation (10) is after multiplication by test function $\eta$ integrated over the whole acoustic domain $\Omega^{a}$ and the application of the Green theorem together with boundary condition (14) yields

$$
\left(\frac{1}{c_{0}^{2}} \frac{\partial^{2} p^{\prime}}{\partial t^{2}}, \eta\right)_{\Omega^{a}}+\left(\nabla p^{\prime}, \nabla \eta\right)_{\Omega^{a}}=-((\operatorname{div} \mathbf{T}), \nabla \eta)_{\Omega^{a}}
$$

Finally, if we restrict us to to finite element space and the solution is sought in form of $p_{h}^{\prime}(t, x)=\sum_{j=1}^{N_{h 2}} \gamma_{j}(t) \eta_{j}(x) \in$ $V_{h} \subset W^{1,2}\left(\Omega^{a}\right)$, it leads to

$$
\mathbb{M}^{a} \ddot{\gamma}+\mathbb{K}^{a} \gamma=\mathbf{b}(t),
$$


where elements of matrices $\mathbb{M}^{a}=\left(m_{i j}^{a}\right), \mathbb{K}^{a}=\left(k_{i j}^{a}\right)$ are given as

$$
m_{i j}^{a}=\left(\frac{1}{c_{0}^{2}} \eta_{i}, \eta_{j}\right)_{\Omega^{a}}, \quad k_{i j}^{a}=\left(\frac{\partial \eta_{i}}{\partial x_{m}}, \frac{\partial \eta_{j}}{\partial x_{m}}\right)_{\Omega^{a}},
$$

and components of vector $\mathbf{b}(t)$ are

$$
b_{i}^{L H}=-\left(\frac{\partial T_{j m}}{\partial x_{m}}, \frac{\partial \eta_{i}}{\partial x_{j}}\right)_{\Omega^{a}},
$$

with upper index ${ }^{L H}$ notifying the Lighthill case.

Repeating the same steps in the case of APE (15) ends with the form as (27), only the right hand side vector $\mathbf{b}(t):=\mathbf{b}^{A P E}(t)=\left(b_{i}^{A P E}\right)$ is different

$$
b_{i}^{A P E}=-\left(\frac{1}{\rho_{0}^{f} c_{0}^{2}}\left(\frac{\partial p_{i c}}{\partial t}+\overline{\mathbf{v}} \cdot \nabla p_{i c}\right), \eta_{i}\right)_{\Omega^{a}} .
$$

In both cases system (27) is discretized in time by the Newmark method.

\section{NUMERICAL RESULTS}

In this section are listed the numerical results of acoustic field generated by flow around an airfoil or in a channel.

\subsection{Numerical results of the flow around an airfoil}

For the flow over an airfoil only the fluid-acoustic coupling was considered and the motion of the structural part was omitted in order to simplify the model. The results are shown for NACA0012 profile under angle of attack $\alpha=$ $5^{\circ}$ with inlet velocity $\mathbf{u}_{\text {In }}=10.0 \mathrm{~m} / \mathrm{s}$ and related Reynold number $\operatorname{Re}=5000$. Time step was chosen as $\Delta t=10^{-6} \mathrm{~s}$. This setting excites the regular separation of flow at the trailing edge as in the case of the von Kárman vortex street as it was expected. The flow separation produces vorticity eddies in the wake behind profile, see Fig. 3.

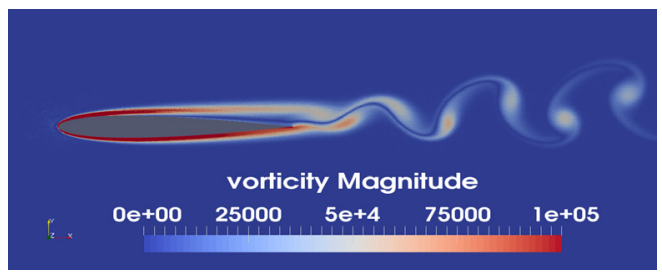

Fig. 3. The characteristic distribution of vorticity.

The sound sources given by (10) and (15) are plotted in Fig. 4. The sound sources in the Lighthill case correspond well with the plotted vorticity, meanwhile the sound sources of APE analogy are closely connected with the flow of pressure depression in the center of each vortex rather than with high velocity gradient at the vinicity of the leading edge.

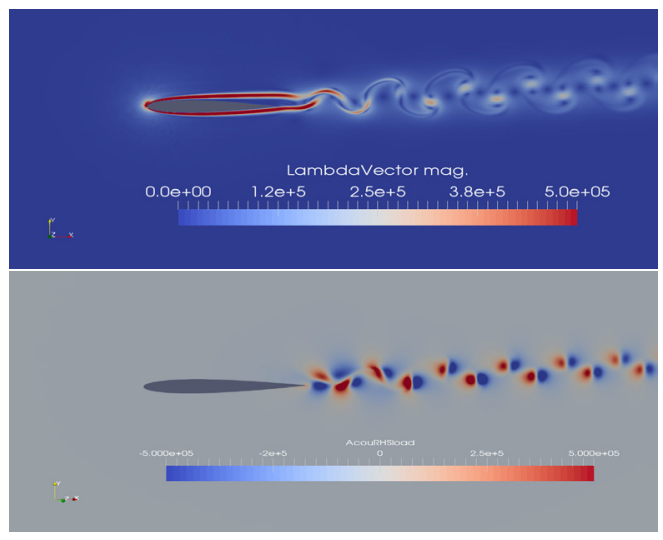

Fig. 4. Top: The distribution of the Lighthill tensor magnitude. Down: The sound source density in the case of APE analogy.

\subsection{Numerical results of FSAI}

The FSI results were achieved by in-house FEM program with implemented P1-bubble/P1 elements, acoustics were computed by academic solver CFS++ from TU Vienna. For the FSI as well as for the transient acoustic simulation the time step was chosen as $2.5 \cdot 10^{-5} \mathrm{~s}$. The four layered vocal fold (VF) model was the same as in [16], but the geometry of channel behind glottis was changed, see schematic figure 5 . The fluid flow was driven by the kinematic pressure difference between inlet and outlet $\Delta p=300 \mathrm{~Pa} \cdot \mathrm{m}^{3} / \mathrm{kg}$. The airflow excited model to vibrate two dominant frequencies equal to first two eigenfrequencies obtained by the modal analysis.

Then the sound sources were computed by Lighthill analogy or by APE, see figure 6 . The structure of the sources are very similar, however the resulting magnitudes of sources are quite different (figures are normalized).

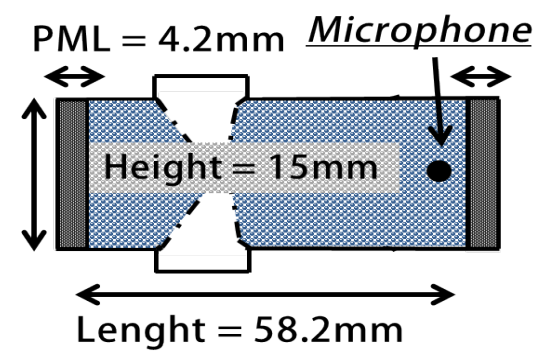

Fig. 5. Dimensions of the vocal fold model with PML blocks at inlet and outlet.

The magnitude difference can be seen on the results of acoustic simulation. The results of Lighthill analogy have overestimated the low frequencies under $600 \mathrm{~Hz}$, the higher frequencies already correspond relatively well to APE, see Fig. 7. The sound at the microphone position could be expected to have the distinct peaks equal to the dominant frequencies of VF motion and higher harmonics, what does not have to coincide with the basic frequencies 
of a specific vowel created by filtering in a vocal tract, see [3]. However our results do not confirm such hypothesis, but the reason can be caused by 2D FSI simulation with overestimation of induced vorticity behind the glottis.

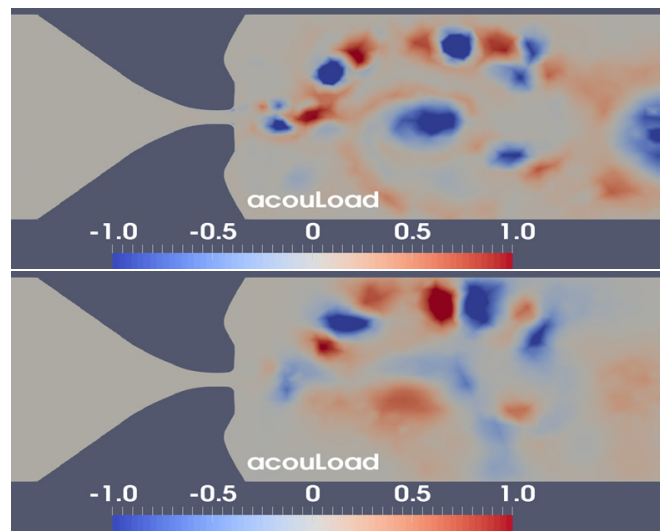

Fig. 6. The sound sources projected onto acoustic grid and normalized to 1 at time $0.24 \mathrm{~s}$. Top: Lighthill analogy. Bottom: APE analogy.

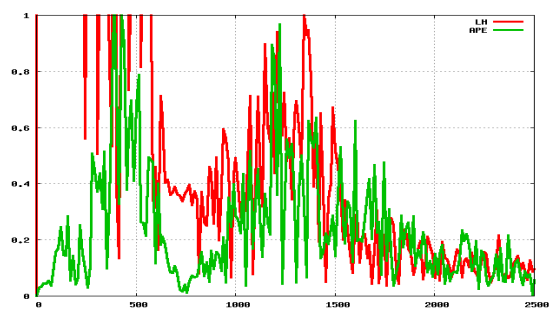

Fig. 7. The frequency spectra of sound pressure level at the microphone position. Lighthill results were normalized to correspond with APE at frequency $1258 \mathrm{~Hz}$.

\section{CONCLUSION}

This contribution presents the mathematical description of the FSAI, where the hybrid partitioned approach was applied. The Lighthill acoustic analogy and APE analogy was used for the determination of the acoustic sources and computation of their propagation through surrounding acoustic domain.

The all considered physical problems are numerically solved by the FEM. The fluid solver was stabilized by SUPG, PSPG and 'div-div' method. The sound sources were computed and interpolated on coarser acoustic grid. PML layer was added to the acoustic domain to a account for free radiation. In the end induced VF vibration by fluid flow was computed with the help of in-house developed program and then the results of sound propagation were obtain by $\mathrm{CFS}++$ solver. The results showed the general applicability of presented methodology for problems of low Mach number flows.

Acknowledgements. Authors acknowledge support from the EU Operational Programme Research, Development and Education, and from the Center of Advanced Aerospace Technology (CZ.02.1.01/0.0/0.0/16_019/0000826), Faculty of Mechanical Engineering, Czech Technical University in Prague.

\section{References}

1. M. De Gennaro, H. Kühnelt, M. Kaltenbacher, in 18th AIAA/CEAS Aeroacoustics Conference (33rd AIAA Aeroacoustics Conference) (2012), p. 2135

2. M. Kaltenbacher, Numerical simulation of mechatronic sensors and actuators: finite elements for computational multiphysics (Springer, 2015)

3. G. Link, M. Kaltenbacher, M. Breuer, M. Döllinger, Computer Methods in Applied Mechanics and Engineering 198, 3321 (2009)

4. M.J. Lighthill, in Proceedings of the Royal Society of London, vol. 211 (The Royal Society, 1952), vol. 211, pp. 564-587

5. J. Hardin, D. Pope, Theoretical and Computational Fluid Dynamics 6(5), 323 (1994)

6. R. Ewert, W. Schröder, Journal of Computational Physics 188(2), 365 (2003)

7. M. Kaltenbacher, M. Escobar, S. Becker, I. Ali, International Journal for Numerical Methods in Fluids 63(9), 1103 (2010)

8. B. Kaltenbacher, M. Kaltenbacher, I. Sim, Journal of Computational Physics 235, 407 (2013)

9. T. Gelhard, G. Lube, M.A. Olshanskii, J.H. Starcke, Journal of Computational and Applied Mathematics 177(2), 243 (2005).

10. M. Feistauer, P. Sváček, J. Horáček, in Fluidstructure Interaction and Biomedical Applications, ed. by T. Bodnár, G.P. Galdi, S. Nečasová (Birkhauser, 2014), pp. 312-393

11. J. Valášek, P. Sváček, J. Horáček, in EFM15 - Experimental Fluid Mechanics 2015, vol. 114, ed. by V. M., D. P. (EPJ Web of Conferences, 2016), vol. 114.

12. M. Braack, P.B. Mucha, Journal of Computational Mathematics 32, 507 (2014)

13. A. Hüppe, Spectral finite elements for acoustic field computation. Ph.D. thesis, Alpen-Adria-Universität Klagenfurt (2012)

14. R.A. Adams, Sobolev Spaces (Academic Press, New York, 1975)

15. A. Curnier, Computational methods in solid mechanics (Springer Netherlands, 1994)

16. J. Valášek, M. Kaltenbacher, P. Sváček, in Topical problems of fluid mechanics 2017, ed. by D. Šimurda, T. Bodnár (Institute of Thermomechanics, AS CR, v.v.i., 2017), pp. 303-312. 